\title{
Detection of the Water Reservoir in a Forming Planetary System
}

\author{
Michiel R. Hogerheijde, ${ }^{1}$ Edwin A. Bergin, ${ }^{2}$ Christian Brinch, ${ }^{1}$ L. Ilsedore Cleeves, ${ }^{2}$ \\ Jeffrey K. J. Fogel, ${ }^{2}$ Geoffrey A. Blake, ${ }^{3}$ Carsten Dominik, ${ }^{4}$ \\ Dariusz C. Lis, ${ }^{5}$ Gary Melnick, ${ }^{6}$ David Neufeld, ${ }^{7}$ Olja Panić, ${ }^{8}$ \\ John C. Pearson, ${ }^{9}$ Lars Kristensen, ${ }^{1}$ Umut A. Yildız, ${ }^{1}$ Ewine F. van Dishoeck ${ }^{1,10}$ \\ ${ }^{1}$ Leiden Observatory, Leiden University, \\ PO Box 9513, 2300 RA Leiden, The Netherlands \\ ${ }^{2}$ Department of Astronomy, University of Michigan, Ann Arbor MI, USA \\ ${ }^{3}$ Div. of Geological and Planetary Sciences, California Institute of Technology, Pasadena CA, USA \\ ${ }^{4}$ Astronomical Institute 'Anton Pannekoek', University of Amsterdam, The Netherlands \\ ${ }^{5}$ Div. of Physics, Mathematics, and Astronomy, California Institute of Technology, Pasadena CA, USA \\ ${ }^{6}$ Harvard-Smithsonian Center for Astrophysics, Cambridge MA, USA \\ ${ }^{7}$ Dept. of Physics and Astronomy, Johns Hopkins University, Baltimore MD, USA \\ ${ }^{8}$ European Southern Observatory, Garching, Germany \\ ${ }^{9}$ Jet Propulsion Laboratory, California Institute of Technology, Pasadena CA, USA \\ ${ }^{10}$ Max-Planck-Institut für Extraterrestrische Physik, Garching, Germany \\ *Accepted for publication in Science.
}

Icy bodies may have delivered the oceans to the early Earth, yet little is known about water in the ice-dominated regions of extra-solar planet-forming disks. The Heterodyne Instrument for the Far-Infrared on-board the Herschel Space Observatory has detected emission lines from both spin isomers of cold water vapor from the disk around the young star TW Hydrae. This water vapor likely originates from ice-coated solids near the disk surface hinting at a water 


\section{ice reservoir equivalent to several thousand Earth oceans in mass. The wa- ter's ortho-to-para ratio falls well below that of solar system comets, suggest- ing that comets contain heterogeneous ice mixtures collected across the entire solar nebula during the early stages of planetary birth.}

Water in the solar nebula is thought to have been frozen out onto dust grains outside $\sim 3$ astronomical units (AU (1)) (2). Stored in icy bodies, this water provided a reservoir for impact delivery of oceans to the Earth (3). In planet-forming disks, water vapor is thought to be abundant only in the hot $(>250 \mathrm{~K})$ inner regions where ice sublimates and gas-phase chemistry locks up all oxygen in $\mathrm{H}_{2} \mathrm{O}$. Emission from hot $(>250 \mathrm{~K})$ water has been detected from several disks around young stars $(4,5)$. In the cold $(\sim 20 \mathrm{~K})$ outer disk water vapor freezes out, evidenced by spectral features of water ice in a few disks $(6,7)$. However, (inter)stellar ultraviolet radiation penetrating the upper disk layers desorbs a small fraction of water ice molecules back into the gas phase $(8)$, suggesting that cold $(<100 \mathrm{~K})$ water vapor exists throughout the radial extent of the disk. The detection of this water vapor would signal the presence of a hidden ice reservoir.

We report detection of ground-state rotational emission lines of both spin isomers of water $\left(J_{K_{A} K_{C}} 1_{10}-1_{01}\right.$ from ortho- $\mathrm{H}_{2} \mathrm{O}$ and $1_{11}-0_{00}$ from para- $\mathrm{H}_{2} \mathrm{O}$ ) from the disk around the premain-sequence star TW Hydrae (TW Hya) using the Heterodyne Instrument for the Far-Infrared (HIFI) spectrometer (9) on-board the Herschel Space Observatory (10) (Fig. 1) (11, 12). TW Hya is a $0.6 \mathrm{M}_{\odot}$ (solar mass), 10-million-year old $\mathrm{T}$ Tauri star (13) $53.7 \pm 6.2 \mathrm{pc}$ away from Earth. Its 196 AU radius disk is the closest protoplanetary disk to Earth with strong gas emission lines. The disk's mass is estimated at $2 \times 10^{-4}$ to $6 \times 10^{-4} \mathbf{M}_{\odot}$ in dust and, using different tracers and assumptions, between $4 \times 10^{-5}$ and $0.06 \mathrm{M}_{\odot}$ in gas (14-16). The velocity widths of the $\mathrm{H}_{2} \mathrm{O}$ lines (0.96 to $1.17 \mathrm{~km} \mathrm{~s}^{-1}$ ) (table $\left.\mathrm{S} 1\right)$ exceed by $\sim 40 \%$ those of cold $\mathrm{CO}$ (14). These correspond to $\mathrm{CO}$ emission from the full $196 \mathrm{AU}$ radius rotating disk inclined at $\sim 7^{\circ}$ with only little $\left(<65 \mathrm{~m} \mathrm{~s}^{-1}\right)$ turbulence (17). The wider $\mathrm{H}_{2} \mathrm{O}$ lines suggest that the water emission extends 
to $\sim 115 \mathrm{AU}$ where the gas orbits the star at higher velocities compared with $196 \mathrm{AU}$.

To quantify the amount of water vapor traced by the detected lines, we performed detailed simulations of the water chemistry and line formation using a realistic disk model matching previous observations $(12,18)$. We adopted a conservatively low dust mass of $1.9 \times 10^{-4} \mathrm{M}_{\odot}$ and, using a standard gas-to-dust mass ratio of 100 , a gas mass of $1.9 \times 10^{-2} \mathrm{M}_{\odot}$. We explored the effects of much lower gas-to-dust ratios. We followed the penetration of the stellar ultraviolet and x-ray radiation into the disk, calculated the resulting photodesorption of water and ensuing gas-phase chemistry including photodissociation, and solved the statistical-equilibrium excitation and line formation. The balance of photodesorption of water ice and photodissociation of water vapor results in an equilibrium column of water $\mathrm{H}_{2} \mathrm{O}$ vapor throughout the disk (Fig. 2). Consistent with other studies (19), we find a layer of maximum water vapor abundance of $0.5 \times 10^{-7}$ to $2 \times 10^{-7}$ relative to $\mathrm{H}_{2}$ at an intermediate height in the disk. Above this layer, water is photodissociated; below it, little photodesorption occurs and water is frozen out, with an ice abundance, set by available oxygen, of $10^{-4}$ relative to $\mathrm{H}_{2}$.

In our model, the 100- to 196-AU region dominates the line emission, which exceeds observations in strength by factors of $5.3 \pm 0.2$ for $\mathrm{H}_{2} \mathrm{O} 1_{10}-1_{01}$ and $3.3 \pm 0.2$ for $\mathrm{H}_{2} \mathrm{O} 1_{11}-0_{00}$. A lower gas mass does not decrease the line intensities, if we assume that the water ice, from which the water vapor derives, formed early in the disk's evolution, before substantial gas loss occurred, and remains frozen on grains. The most plausible explanation involves a difference in the relative location of small, bare grains regulating the ultraviolet radiative transport and larger, ice-carrying grains. Differential settling of large grains relative to small grains moves much of the ice reservoir below the reach of the ultraviolet radiation, resulting in less water vapor and weaker lines. Our model matches the observations if only $12 \%$ of the original ice content remains above this line (20). A radially increasing degree of settling of icy grains explains the observed $\mathrm{H}_{2} \mathrm{O}$ line widths. 
The detected water vapor, resulting from photodesorption, implies an ice reservoir in the giant planet formation zone and beyond. In our simulations the $7.3 \times 10^{21} \mathrm{~g}$ of detected water vapor (equivalent to 0.005 times the mass of Earth's oceans) originate from a total ice reservoir of $9 \times 10^{27} \mathrm{~g}$ (or several thousands of Earth's oceans) throughout the disk. The size of this reservoir is tied to the dust mass contained in the disk, for which we adopt a conservatively low value. Although the ice reservoir is only observed indirectly, no known mechanism can remove it from the regions probed by Herschel. Any smaller ice reservoir implies the corresponding absence of elemental oxygen that efficiently reforms water ice on the grains.

The detection of both spin isomers of water vapor allows its ortho-to-para ratio (OPR) to be derived, because our simulations indicate that the lines are optically thin. An OPR of $0.77 \pm$ 0.07 matches our observations (12). This value is much lower than the OPR range of 2 to 3 observed for solar system comets $(21)$. It is common practice to associate the OPR with a spin temperature $T_{\text {spin }}$ at which a Boltzmann distribution reproduces the ratio of spin isomers. Our derived OPR corresponds to $T_{\text {spin }}=13.5 \pm 0.5 \mathrm{~K}$, whereas the range for solar system comets yields a $T_{\text {spin }}$ of $>20 \mathrm{~K}$.

Radiative conversion between spin isomers is not allowed in the gas phase, preserving the OPR for long timescales. Gas-phase formation of water occurs through exothermic reactions leading to an OPR of 3. On grains, water forms and survives at low temperatures, and it is tempting to equate $T_{\text {spin }}$ with the grain temperature. However, the energetics of water formation and ortho-to-para exchange on grains are poorly understood (22), and the water OPR may be changed by photodesorption. This process starts by dissociating water to $\mathrm{H}$ and $\mathrm{OH}$ in the ice, and continues with the energetic $\mathrm{H}$ kicking out a neighboring $\mathrm{H}_{2} \mathrm{O}$ molecule from the ice matrix, or with the $\mathrm{H}$ and $\mathrm{OH}$ recombining in the ice to form $\mathrm{H}_{2} \mathrm{O}$ with sufficient internal energy to sublimate (23). The latter route drives the OPR to at least unity, implying an even lower original ice OPR, to yield a resulting OPR of 0.77 . Cometary volatiles are released through 
thermal sublimation and their measured OPRs are interpreted to reflect the OPR of their ice constituents. Equating $T_{\text {spin }}$ with the physical temperature of the grain on which the ice formed is supported by the similarity of measured $T_{\text {spin }}$ of $\mathrm{NH}_{3}$ and $\mathrm{H}_{2} \mathrm{O}$ in several individual solar system comets (24).

Solar system comets consist of a heterogeneous mixture of ices and solids, likely assembled in the giant planet formation zone by mixing local material with material that drifted in from larger radii (25). Our water vapor observations probe cold, ice-coated precometary grains residing beyond $>50 \mathrm{AU}$ representing the bulk of the latter material. The presence in comets of crystalline silicates, requiring formation temperatures $>800 \mathrm{~K}(26)$, together with $\mathrm{CO}$ and $\mathrm{H}_{2} \mathrm{O}$ ices that condense at 20 to $100 \mathrm{~K}$, argues for transport of hot material from near the star to the icy outer regions of the solar nebula (27). Provided that spin temperatures reflect formation histories, the different $T_{\text {spin }}$ inferred for the water ice in TW Hya $(<13 \mathrm{~K})$ and solar system comets ( $>20 \mathrm{~K}$ ) indicates a similar mixing of volatiles throughout the entire solar nebula, blending water formed at $>50 \mathrm{~K}$ and an OPR of 3, with water formed at 10 to $20 \mathrm{~K}$ and $\mathrm{OPR}<1$ probed by

our observations. In this case, the range of $T_{\text {spin }}$ values of the cometary inventory reflects the stochastic nature of transport and mixing.

Our Herschel detection of cold water vapor in the outer disk of TW Hya demonstrates the presence of a considerable reservoir of water ice in this protoplanetary disk, sufficient to form several thousand Earth oceans worth of icy bodies. Our observations only directly trace the tip of the iceberg of 0.005 Earth oceans in the form of water vapor.

\section{References and Notes}

1. One astronomical unit (AU) is the mean distance between Earth and the Sun of $1.49598 \times$ $10^{11} \mathrm{~m}$. 
2. C. Hayashi, Progress of Theoretical Physics Supplement 70, 35 (1981).

3. T. Matsui, Y. Abe, Nature 322, 526 (1986).

4. K. M. Pontoppidan, C. Salyk, G. A. Blake, H. U. Käufl, Astrophys. J. 722, L173 (2010).

5. J. S. Carr, J. R. Najita, Astroph. J. 733, 102 (2011).

6. H. Terada, et al., Astrophys. J. 667, 303 (2007).

7. M. Honda, et al., Astrophys. J. 690, L110 (2009).

8. C. Dominik, C. Ceccarelli, D. Hollenbach, M. Kaufman, Astrophys. J. 635, L85 (2005).

9. T. de Graauw, et al., Astron. Astrophys. 518, L6 (2010).

10. G. L. Pilbratt, et al., Astron. Astrophys. 518, L1 (2010).

11. Water in Earth's atmosphere obstructs ground-based detection of cold water vapor in planetforming disks. Although Herschel cannot spatially resolve even the closest disk in water ground-state emission lines, HIFI spectrally resolves the $\mathrm{H}_{2} \mathrm{O}$ line profiles. Comparison with previous spectrally and spatially resolved observations of $\mathrm{CO}$ confirms the disk origin of the $\mathrm{H}_{2} \mathrm{O}$ lines.

12. Materials and methods are available as supporting material on Science online.

13. R. A. Webb, et al., Astrophys. J. 512, L63 (1999).

14. J. H. Kastner, B. Zuckerman, D. A. Weintraub, T. Forveille, Science 277, 67 (1997).

15. N. Calvet, et al., Astrophys. J. 568, 1008 (2002).

16. U. Gorti, D. Hollenbach, J. Najita, I. Pascucci, Astroph. J. 735, 90 (2011). 
17. A. M. Hughes, D. J. Wilner, S. M. Andrews, C. Qi, M. R. Hogerheijde, Astrophys. J. 727, 85 (2011).

18. W. Thi, et al., Astron. Astroph. 518, L125 (2010).

19. P. Woitke, W. Thi, I. Kamp, M. R. Hogerheijde, Astron. Astrophys. 501, L5 (2009).

20. R. Meijerink, K. M. Pontoppidan, G. A. Blake, D. R. Poelman, C. P. Dullemond, Astrophys. J. 704, 1471 (2009).

21. B. P. Bonev, et al., Astrophys. J. 661, L97 (2007).

22. H.-H. Limbach, et al., Chem. Phys. Chem. 7, 551 (2006).

23. S. Andersson, E. F. van Dishoeck, Astron. Astrophys. 491, 907 (2008).

24. Y. Shinnaka, et al., Astroph. J. 729, 81 (2011).

25. S. J. Weidenschilling, Mon. Not. R. Astron. Soc. 180, 57 (1977).

26. S. A. Sandford, et al., Science 314, 1720 (2006).

27. D. H. Wooden, Space Science Reviews 138, 75 (2008).

Herschel is an European Space Agency space observatory with science instruments provided by European-led principal investigator consortia and with important participation from NASA. This work was partially supported by Nederlandse Organisatie voor Wetenschappelijk Onderzoek grant 639.042.404, NSF grant 0707777 and, as part of the NASA Herschel HIFI guaranteed time program, by NASA. The data presented here are archived at the Herschel Science Archive, http://archives.esac.esa.int/hda/ui/, under OBSID 1342198337 and 1342201585.

\section{Supporting Online Material}


Materials and Methods

Table S1

References (28-39) 


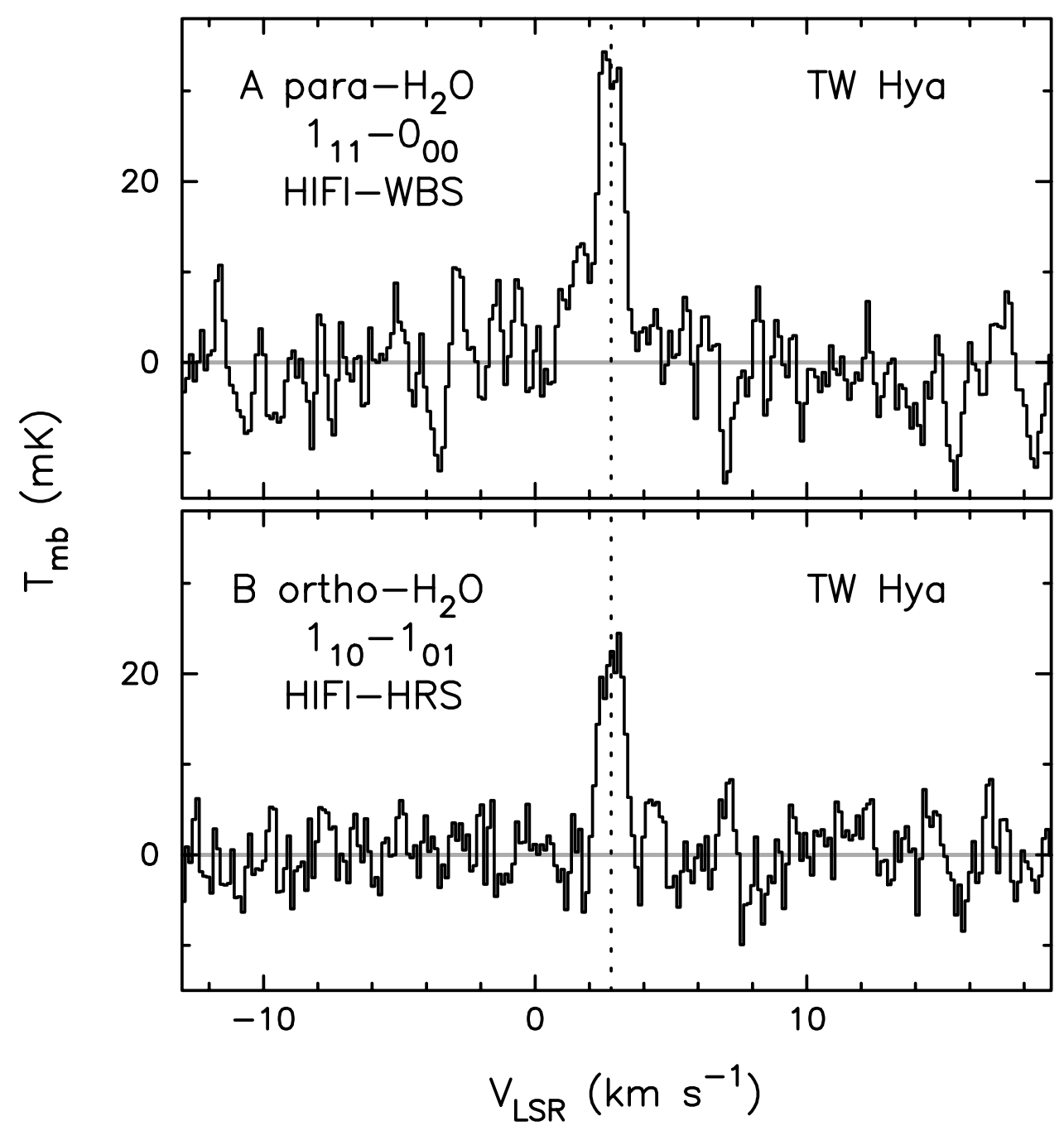

Figure 1: Spectra of para- $\mathrm{H}_{2} \mathrm{O} 1_{11}-0_{00}(\mathbf{A})$ and ortho- $\mathrm{H}_{2} \mathrm{O} 1_{10}-1_{01}(\mathbf{B})$ obtained with HIFI on the Herschel Space Observatory toward the protoplanetary disk around TW Hya after subtraction of the continuum emission. The vertical dotted lines show the system's velocity of $+2.8 \mathrm{~km} \mathrm{~s}^{-1}$ relative to the Sun's local environment (local standard of rest). 

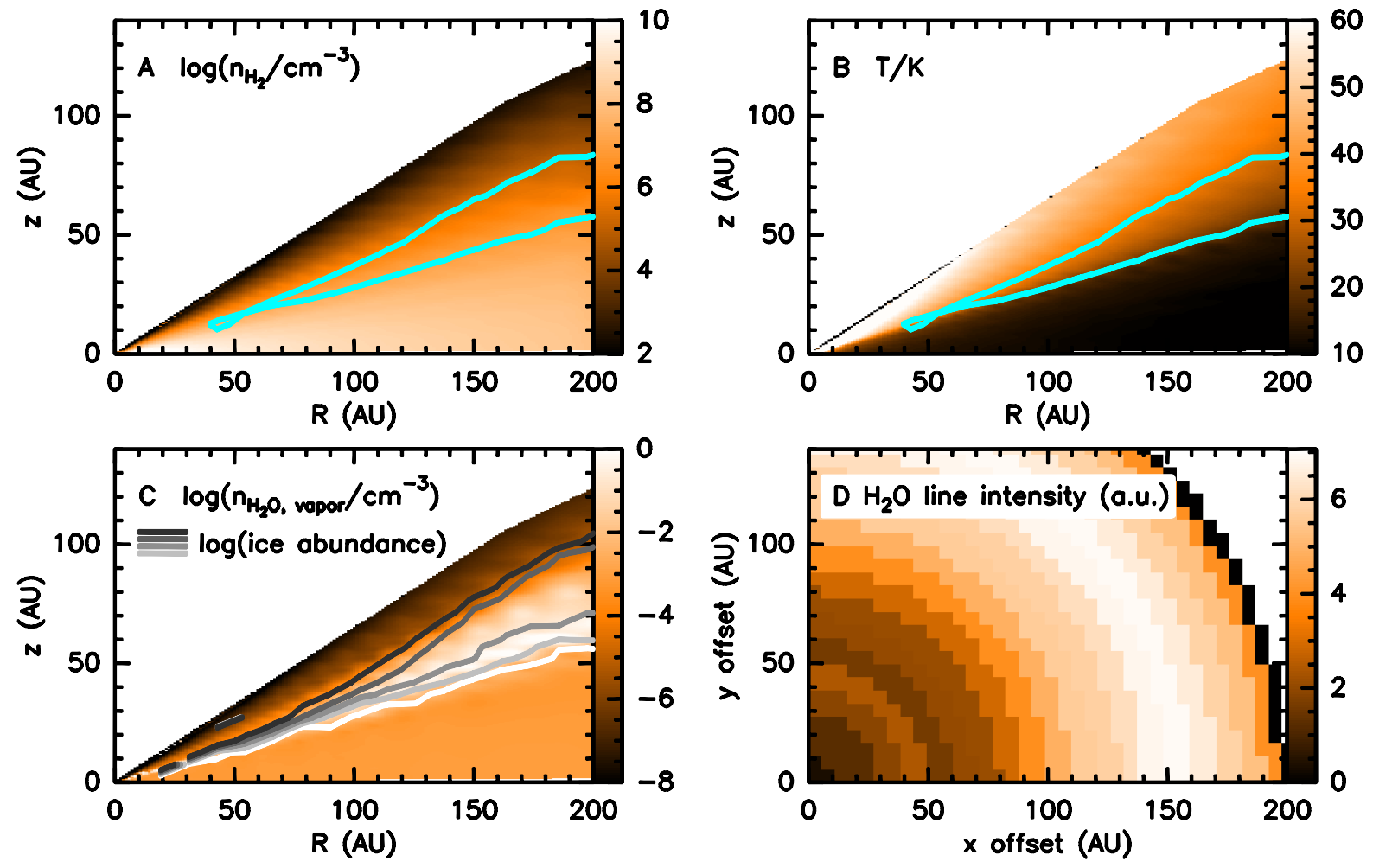

Figure 2: Adopted model for the TW Hya protoplanetary disk: (A) $\mathrm{H}_{2}$ number density, (B) dust temperature , $(\mathbf{C})$ the number density of water vapor molecules and contours of volumeaveraged water ice abundance decreasing from white to black as $2 \times 10^{-4}, 2 \times 10^{-5}, 2 \times 10^{-6}$, $2 \times 10^{-7}$, and $2 \times 10^{-8}$, relative to $\mathrm{H}_{2}$, and (D) one quadrant of the resulting water emission line intensity from the near face-on disk, in arbitrary units. In (A) and (B), the blue contour delineates the layer of maximum water vapor abundance. 


\section{Supporting Online Material}

\section{Materials and Methods}

\section{Observations and data reduction}

We observed TW Hya $\left(\alpha_{2000}=11^{\mathrm{h}} 01^{\mathrm{m}} 51.91^{\mathrm{s}}, \delta_{2000}=-34^{\circ} 42^{\prime} 17.0^{\prime \prime}\right)$ with the Heterodyne Instrument for the Far-Infrared (HIFI) onboard the Herschel Space Observatory in double beamswitch mode with a throw of 3.0', as part of the 'Water in Star-Forming Regions with Herschel' (WISH) key program (28). On 2010 June 15 the instrument observed the $\mathrm{H}_{2} \mathrm{O} 1_{10}-1_{01}$ line at $556.936002 \mathrm{GHz}$ using receiver band $\mathrm{lb}$ and a local oscillator tuning of $551.895 \mathrm{GHz}(\mathrm{OB}-$ SID 1342198337). A total on-source integration time of $181 \mathrm{~min}$ was obtained and system temperatures were $75-95 \mathrm{~K}$. On 2010 July 17 the instrument observed the $\mathrm{H}_{2} \mathrm{O} 1_{11}-0_{00}$ line at $1113.342964 \mathrm{GHz}$ with receiver band $4 \mathrm{~b}$ and a Local Oscillator tuning of $1108.221 \mathrm{GHz}$ (OBSID 1342201585). A total on-source integration of 326 min was obtained with system temperatures of 360-400 K.

The data were recorded in the Wide-Band Spectrometer (WBS) which covers $4.4 \mathrm{GHz}$ with 1.1 $\mathrm{MHz}$ resolution. At the observing frequencies of 557 and $1113 \mathrm{GHz}$ this corresponds to 0.59 and $0.30 \mathrm{~km} \mathrm{~s}^{-1}$, respectively. Simultaneously, the data were recorded in the High-Resolution Spectrometer (HRS) which covers $230 \mathrm{MHz}$ at a resolution of $0.25 \mathrm{MHz}(0.13$ and $0.067 \mathrm{~km}$ $\mathrm{s}^{-1}$ at the observing frequencies of 557 and $1113 \mathrm{GHz}$, respectively). Both horizontal and vertical linear polarizations were measured. The relative offsets of the respective polarization channels from our pointing center were $3.3^{\prime \prime}$ at $556 \mathrm{GHz}$ and $1.8^{\prime \prime}$ at $1113 \mathrm{GHz}$, in both cases much smaller than the beams of $38.1^{\prime \prime}$ and $19.1^{\prime \prime}$, respectively, and not relevant for our analysis.

The in-orbit calibration system converted the raw data to the $T_{\mathrm{A}}^{*}$ (antenna temperature) intensity scale. Further conversion to the main-beam antenna temperature scale $T_{\mathrm{mb}}$ was done using a beam efficiency of 0.76 at both observing frequencies, accurate to $10 \%$. The data were reduced using HIPE v4.0.0 (1113 GHz) and v 4.6.0 (556 GHz), and exported to the CLASS software package for further analysis. The velocity accuracy of the final data product is better than a few $\mathrm{m} \mathrm{s}^{-1}$.

After averaging together the horizontal- and vertical-polarization measurements, resulting noise levels are $1.6 \mathrm{mK}$ per $0.27 \mathrm{~km} \mathrm{~s}^{-1}$ WBS channel at $557 \mathrm{GHz}, 4.2 \mathrm{mK}$ per $0.065 \mathrm{~km} \mathrm{~s}^{-1}$ HRS channel at $557 \mathrm{GHz}, 4.95 \mathrm{mK}$ per $0.135 \mathrm{~km} \mathrm{~s}^{-1}$ WBS channel at $1113 \mathrm{GHz}$, and $14.3 \mathrm{mK}$ per $0.032 \mathrm{~km} \mathrm{~s}^{-1} \mathrm{HRS}$ channel at $1113 \mathrm{GHz}$. Throughout the long integration, the noise was confirmed to decrease proportional to (time) $)^{-0.5}(29)$.

Linear baselines were fit using a $50 \mathrm{~km} \mathrm{~s}^{-1}$ interval centered on the source velocity, but excluding a $5 \mathrm{~km} \mathrm{~s}^{-1}$ interval around the emission lines. This yielded continuum levels of 
$5.60 \pm 0.05$ at $557 \mathrm{GHz}$ and $13.2 \pm 0.1 \mathrm{Jy}$ at $1113 \mathrm{GHz}$, which compare well with measured fluxes in the literature (30). After subtraction of the continuum level, single Gaussian profiles were fitted to the clearly visible lines yielding values for the Local Standard of Rest Velocity, $V_{\mathrm{LSR}}$, of the lines, their Full Width at Half Maximum, their peak intensity and their integrated intensity (Table S1).

\section{Physical and chemical model of the TW Hya disk}

The adopted physical model for the TW Hya disk is based on recent modeling of its full spectral energy distribution including Spitzer and Herschel data (18), with parameters similar to those that reproduce a wide set of observations including gas emission lines (16). It has a dust mass of $1.9 \times 10^{-4} \mathrm{M}_{\odot}$ in $<1 \mathrm{~mm}$ particles, and a gas mass of $1.9 \times 10^{-2} \mathrm{M}_{\odot}$ obtained by assuming a gas-to-dust mass ratio of 100 . The adopted disk dust mass is a lower limit, containing only particles $<1 \mathrm{~mm}$ and employing a close-to maximum emissivity to turn continuum flux into mass: other mass estimates are four times larger (in $<1 \mathrm{~mm}$ particles), or as much as 14 times larger if significant grain growth (as expected) is included and 93\% of the mass is $>1 \mathrm{~mm}(18)$. The gas mass is near the high end of the inferred gas masses in the literature of $4 \times 10^{-5}$ $0.06 \mathrm{M}_{\odot}(14,16,18)$. This range reflects the uncertainty in translating observations into gasmass estimates. The low end of the range corresponds to taking $\mathrm{CO}$ observations, assuming an abundance of $10^{-4}$ with respect to $\mathrm{H}_{2}$, and ignoring any freeze out (14). The high end is derived from dust continuum observations and a standard gas-to-dust ratio of 100 (15), but is found to reproduce gas-line observations including $\mathrm{CO}$ when more realistic chemical conditions are adopted (16). We will explore the effects of gas masses lower than the $0.02 \mathrm{M}_{\odot}$ from our baseline model.

The disk's outer radius is $196 \mathrm{AU}$, and its surface density outside a radius of $4 \mathrm{AU}$ is given by $\Sigma(R)=1.4 \times 10^{-2}(R / 100 \mathrm{AU})^{-1.0} \mathrm{~g} \mathrm{~cm}^{-2}$. Inside $4 \mathrm{AU}$ the surface density is much lower, but we can ignore this region in our modeling. The vertical density distribution is characterized by an exponential scale height $H(R)=10(R / 100 \mathrm{AU})^{1.2} \mathrm{AU}$ and a density profile $\rho(z, R) \propto$ $\exp \left(-z^{2} / 2 H^{2}\right)$. The dust particles have a size distribution $N(a) \propto a^{-3.4}$ between $a_{\min }=0.03$ $\mu \mathrm{m}$ and $a_{\max }=10 \mathrm{~cm}$, and a mean density of $3.5 \mathrm{~g} \mathrm{~cm}^{-3}$. Gas and dust are assumed to be well mixed throughout the disk. The dust temperature is calculated for a stellar effective temperature of $4000 \mathrm{~K}$ and a luminosity of $0.23 \mathrm{~L}_{\odot}$ with the RADMC code (31), which yields a result identical to the original model. The gas and dust temperatures are assumed to be identical.

The penetration of the stellar ultraviolet radiation, both continuum and $\mathrm{Ly} \alpha$, is calculated for the defined density structure, adopting the measured stellar spectrum and ultraviolet dust scattering properties from (32). Subsequently, the chemical composition throughout the disk is calculated following the methods of (33) including the effects of gas-phase reactions, thermal 
desorption, desorption by ultraviolet radiation (34), cosmic-ray induced ultraviolet photons, and photodissociation by ultraviolet radiation. X-ray propagation and dissociation of $\mathrm{H}_{2}$ by other species is also included in the calculation, but do not influence our results. Cosmic ray induced photodesorption provides the only source of water vapor in the disk interior, leading to a low base-level of water vapor abundance of $\sim 10^{-10}$ relative to $\mathrm{H}_{2}(35)$. Our simulations do not treat the disk structure and water chemistry in the inner disk $(<5 \mathrm{AU})$ correctly, but in the large Herschel beam these regions contribute only negligible amounts of line flux. Except for the inner several AU, the temperatures in the disk are everywhere well below $200 \mathrm{~K}$, excluding the efficient formation of water in the gas phase, leaving photodesorption of water ice as the sole source of water vapor to explain the Herschel observations.

This baseline model contains a total water ice reservoir of 6300 Earth Oceans (equivalent with $9 \times 10^{27} \mathrm{~g}$, with one Earth Ocean containing $1.5 \times 10^{24} \mathrm{~g}$ of water), mostly as ice frozen out onto dust grains. This ice reservoir is a rough estimate at best. It is based on a low dust mass of $1.9 \times 10^{-4} \mathrm{M}_{\odot}$. It assumes that all water ice thought to be present in the protostellar core from which TW Hya formed was either retained or efficiently reformed from gas-phase oxygen on the dust grains. We adopt an oxygen abundance of $3.5 \times 10^{-4}$ relative to $\mathrm{H}$, with $70 \%$ locked up in water and $30 \%$ in CO (36). As such, our model may overestimate the ice reservoir if the disk is formed depleted in elemental oxygen, because the amount of water ice scales linearly with the oxygen abundance. This may be the case, if the water ice formed after the disk underwent significant loss of its gas mass. For example, if the disk lost $90 \%$ of its gas mass before the water ice formed, the water ice reservoir would be lowered by a corresponding factor.

\section{Excitation of the water molecule and formation of the emis- sion lines}

Most water vapor is present at $\mathrm{H}_{2}$ number densities of a few times $10^{6} \mathrm{~cm}^{-3}$ and temperatures of 25-35 K. This necessitates a full calculation of the statistical equilibrium populations of the involved levels, because these values are below the critical density and upper level energies for the transitions. The column density of water molecules, and resulting line opacities, further necessitates a full calculation of the transport of line photons including absorption and resonant scattering. We calculated the statistical equilibrium excitation and emission line formation with the LIME code (37). Only collisions with $\mathrm{H}_{2}$ are included; at a relative fraction of $<10^{-7}$ relative to $\mathrm{H}_{2}$ in the region of interest, electrons do not contribute significantly to the excitation. Collisional rate coefficients were taken from $(38,39)$ and an $\mathrm{H}_{2}$ ortho-to-para ratio of 3.0 was adopted. The water vapor ortho-to-para ratio in our standard model is characterized by a spin 
temperature equal to the local dust temperature.

Our simulations show that the excitation of the relevant energy levels is subthermal, as expected for densities lower than the critical densities of the transitions of $\sim 2 \times 10^{7} \mathrm{~cm}^{-3}$ for $\mathrm{H}_{2} \mathrm{O} 1_{10}-1_{01}$ and $\sim 2 \times 10^{8} \mathrm{~cm}^{-3}$ for $\mathrm{H}_{2} \mathrm{O} 1_{11}-0_{00}$. In the standard model the mean free path of the photons is small compared to the thickness of the layer with abundant water vapor. Still, the emission is effectively optically thin, because of the significant chance of resonant scattering compared to photon absorption in this excitation regime. Crucially, this means that our observations are sensitive to the total amount of water vapor as opposed to fully optically thick lines. However, it also means that the calculated emission of the individual lines depends sensitively on the location of the peak water abundance, since from this follows the surrounding $\mathrm{H}_{2}$ number density and therefore the excitation conditions. The sensitivity of the excitation on the gas temperature is much smaller.

Our standard model overestimates the water fluxes by factors 3-5 compared to the observations. The line strengths can be reduced if the collisional excitation is decreased or if the total amount of water vapor is lowered. We first explore the dependency of our results on the collisional excitation, through the effects of the overall $\mathrm{H}_{2}$ density and the $\mathrm{H}_{2}$ ortho-to-para ratio (OPR). The results of our standard model do not change significantly when we increase the $\mathrm{H}_{2}$ density or change the $\mathrm{H}_{2}$ OPR from 3 to 0.1 . The latter corresponds to an $\mathrm{H}_{2} \mathrm{OPR}$ in thermal equilibrium at a temperature of $\sim 35 \mathrm{~K}$ as appropriate for the layer of maximum water abundance, and represents a lower limit to the $\mathrm{H}_{2}$ OPR. The lack of dependency can be explained by the small mean free path of the water line photons in the standard model, which contributes to the excitation of the energy levels. Only when the $\mathrm{H}_{2}$ density is lowered by an order of magnitude does the excitation of the water molecules and the emergent line strengths decrease. Within our model, decreasing the $\mathrm{H}_{2}$ density corresponds to increasing the height of the layer of maximum water abundance. Since the latter is set by the vertical distribution of the dust, this corresponds to increasing the height of the dust. However, the dust is supported vertically by the gas pressure, and increasing the vertical height of the dust is therefore not allowed. Decreasing the height of the dust is allowed, as happens when all grains, independent of grain size, decouple partially from the gas and settle uniformly to the midplane. This moves the layer of maximum water abundance to a region of increased $\mathrm{H}_{2}$ density, but does not affect the line strengths significantly because the increased excitation is balanced by increased line opacity.

Reducing the total gas mass of the disk does not decrease the strength of the water emission lines. The reason for this is two-fold. First, we assume that the water ice, from which the water vapor derives through photodesorption, was formed on the grains early in the disk's history, before any significant gas loss occurred, and remains unchanged. Therefore, even for low disk gas masses, the column density of water vapor is unchanged. Secondly, as the gas density drops, the ice-carrying grains, which are vertically supported by the gas pressure, must settle to 
the midplane. As a result, we expect the $\mathrm{H}_{2}$ density in the layer of water vapor to remain similar as the vertical height of the layer is reduced. The collisional excitation of the water vapor molecules therefore is not changed significantly. Together, these two effects lead to essentially unchanged water vapor emission line strengths and unchanged total amount of ice even if the gas-to-dust ratio is lowered. This reasoning rests crucially on the assumption that the water ice formed at an original gas-to-dust ratio of 100. If the ice formed after $90 \%$ of the gas has been lost, the resulting ten times smaller ice reservoir can explain our observations. However, there are no likely scenarios in which the water ice starts to form only at such an advanced stage of the disk's evolution.

The lack of viable scenarios to reduce the intensities of the water lines by changing the $\mathrm{H}_{2}$ density, its OPR, or the total gas mass leaves as our only option a decrease of the total amount of water vapor to match the observations. Since the water vapor derives from photodesorption of ice, this means that the amount of water ice in the regions affected by ultraviolet radiation needs to be reduced. We calculated models where the abundance of ortho-water and para-water were reduced uniformly throughout the disk with separate factors until the emerging line intensities match the observations. Unlike our standard model, $T_{\text {spin }}$ now no longer is assumed equal to the dust temperature but instead is constant throughout the disk. Compared to the total amount of water vapor predicted by the standard model, a model where $5.4 \pm 0.2 \%$ is present as orthowater and $7.0 \pm 0.3 \%$ as para-water reproduced the observed line intensities (i.e., a water OPR of $0.77 \pm 0.07)$. The error bars reflect only the statistical uncertainty due to the measurement error in the observed line intensities; systematic uncertainties are discussed below. This corresponds to a reduction to $12 \%$ of the ice content in the regions affected by the ultraviolet radiation, although the size of the total ice reservoir does not change. At these low abundances, the disk becomes fully optically thin to the water line photons.

We finally investigated the dependency of the derived water OPR at these reduced abundances on the water excitation through collisions with $\mathrm{H}_{2}$, including the effect of the $\mathrm{H}_{2} \mathrm{OPR}$. Because the critical density of the para- $\mathrm{H}_{2} \mathrm{O} 1_{11}-0_{00}$ line is higher than that of the ortho- $\mathrm{H}_{2} \mathrm{O}$ $1_{10}-1_{01}$ line, increasing the $\mathrm{H}_{2}$ density increases the para- $\mathrm{H}_{2} \mathrm{O}$ line faster than the ortho- $\mathrm{H}_{2} \mathrm{O}$ line, which decreases the water OPR derived from the observed line ratio. A factor of 10 increase in the $\mathrm{H}_{2}$ density increases the derived water OPR to 2.0. Such an increase in $\mathrm{H}_{2}$ density corresponds to significant uniform settling of all dust, both large and small, and the associated layer of maximum water vapor abundance, for which there is no observational evidence in the TW Hya disk at the relevant radii. It would furthermore require an even larger degree of differential settling of large, icy grains with respect to small grains to further reduce the water vapor abundance in order to reproduce the observed line strengths. No degree of uniform settling can yield a derived water OPR of 3 .

In the optically thin limit appropriate for the disk model with reduced water vapor abun- 
dance, reducing the $\mathrm{H}_{2}$ OPR from 3 to 0.1 has an effect comparable to lowering the $\mathrm{H}_{2}$ density since para- $\mathrm{H}_{2}$ has lower collision rates with water compared to ortho- $\mathrm{H}_{2}$. Because of the differences in collision rates between ortho-water and para-water, the derived water OPR increases to 1.3 for an $\mathrm{H}_{2}$ OPR of 0.1. Thus, our conclusion is robust that the OPR in the TW Hya disk is much lower than the range of 2-3 measured for Solar System comets.

From these considerations we conclude that the most likely explanation of our Herschel detection of the emission lines of water vapor from the TW Hya disk, is a situation where only $12 \%$ of the original ice content remains in the regions affected by UV irradiation and the remainder has settled to lower depths. The ortho-to-para ratio of the water vapor, and by extension the ice reservoir, is as low as 0.77 to reproduce the observed ratio of the $1_{11}-0_{00}$ and $1_{10}-1_{01}$ lines.

Table S1. Observed line parameters.

\begin{tabular}{lcccc}
\hline Transition & $\begin{array}{c}V_{\mathrm{LSR}} \\
\left(\mathrm{km} \mathrm{s}^{-1}\right)\end{array}$ & $\begin{array}{c}\text { FWHM } \\
\left(\mathrm{km} \mathrm{s}^{-1}\right)\end{array}$ & $\begin{array}{c}\int T_{\mathrm{mb}} d V \\
\left(\mathrm{mK} \mathrm{km} \mathrm{s}^{-1}\right)\end{array}$ & $\begin{array}{c}F_{\text {line }} \\
\left(10^{-19} \mathrm{~W} \mathrm{~m}^{-2}\right)\end{array}$ \\
\hline $\mathrm{H}_{2} \mathrm{O} 1_{11}-0_{00}$ & $2.73 \pm 0.08$ & $1.2 \pm 0.2$ & $44.6 \pm 2.8$ & $3.07 \pm 0.19$ \\
$\mathrm{H}_{2} \mathrm{O} 1_{10}-1_{01}$ & $2.84 \pm 0.04$ & $0.96 \pm 0.07$ & $25.2 \pm 1.1$ & $3.46 \pm 0.15$ \\
\hline
\end{tabular}

\section{References and Notes}

1. One astronomical unit (AU) is the mean distance between Earth and the Sun of $1.49598 \times$ $10^{11} \mathrm{~m}$.

2. C. Hayashi, Progress of Theoretical Physics Supplement 70, 35 (1981).

3. T. Matsui, Y. Abe, Nature 322, 526 (1986).

4. K. M. Pontoppidan, C. Salyk, G. A. Blake, H. U. Käufl, Astrophys. J. 722, L173 (2010).

5. J. S. Carr, J. R. Najita, Astroph. J. 733, 102 (2011).

6. H. Terada, et al., Astrophys. J. 667, 303 (2007).

7. M. Honda, et al., Astrophys. J. 690, L110 (2009).

8. C. Dominik, C. Ceccarelli, D. Hollenbach, M. Kaufman, Astrophys. J. 635, L85 (2005).

9. T. de Graauw, et al., Astron. Astrophys. 518, L6 (2010).

10. G. L. Pilbratt, et al., Astron. Astrophys. 518, L1 (2010). 
11. Water in Earth's atmosphere obstructs ground-based detection of cold water vapor in planetforming disks. Although Herschel cannot spatially resolve even the closest disk in water ground-state emission lines, HIFI spectrally resolves the $\mathrm{H}_{2} \mathrm{O}$ line profiles. Comparison with previous spectrally and spatially resolved observations of $\mathrm{CO}$ confirms the disk origin of the $\mathrm{H}_{2} \mathrm{O}$ lines.

12. Materials and methods are available as supporting material on Science online.

13. R. A. Webb, et al., Astrophys. J. 512, L63 (1999).

14. J. H. Kastner, B. Zuckerman, D. A. Weintraub, T. Forveille, Science 277, 67 (1997).

15. N. Calvet, et al., Astrophys. J. 568, 1008 (2002).

16. U. Gorti, D. Hollenbach, J. Najita, I. Pascucci, Astroph. J. 735, 90 (2011).

17. A. M. Hughes, D. J. Wilner, S. M. Andrews, C. Qi, M. R. Hogerheijde, Astrophys. J. 727, 85 (2011).

18. W. Thi, et al., Astron. Astroph. 518, L125 (2010).

19. P. Woitke, W. Thi, I. Kamp, M. R. Hogerheijde, Astron. Astrophys. 501, L5 (2009).

20. R. Meijerink, K. M. Pontoppidan, G. A. Blake, D. R. Poelman, C. P. Dullemond, Astrophys. J. 704, 1471 (2009).

21. B. P. Bonev, et al., Astrophys. J. 661, L97 (2007).

22. H.-H. Limbach, et al., Chem. Phys. Chem. 7, 551 (2006).

23. S. Andersson, E. F. van Dishoeck, Astron. Astrophys. 491, 907 (2008).

24. Y. Shinnaka, et al., Astroph. J. 729, 81 (2011).

25. S. J. Weidenschilling, Mon. Not. R. Astron. Soc. 180, 57 (1977).

26. S. A. Sandford, et al., Science 314, 1720 (2006).

27. D. H. Wooden, Space Science Reviews 138, 75 (2008).

28. E. F. van Dishoeck, et al., Pub. Astron. Soc. Pacific 123, 138 (2011).

29. E. A. Bergin, et al., Astron. Astroph. 521, L33 (2010).

30. C. Qi, et al., Astroph. J. 636, L157 (2006).

31. C. P. Dullemond, C. Dominik, Astron. Astroph. 417, 159 (2004).

32. J. C. Weingartner, B. T. Draine, Astroph. J. 548, 296 (2001).

33. J. K. J. Fogel, T. J. Bethell, E. A. Bergin, N. Calvet, D. Semenov, Astroph. J. 726, 29 (2011). 
34. K. I. Öberg, H. Linnartz, R. Visser, E. F. van Dishoeck, Astrophys. J. 693, 1209 (2009).

35. C. J. Shen, J. M. Greenberg, W. A. Schutte, E. F. van Dishoeck, Astron. Astrophys. 415, 203 (2004).

36. R. Visser, E. F. van Dishoeck, S. D. Doty, C. P. Dullemond, Astron. Astroph. 495, 881 (2009).

37. C. Brinch, M. R. Hogerheijde, Astron. Astroph. 523, A25 (2010).

38. A. Faure, et al., Astron. Astroph. 472, 1029 (2007).

39. M.-L. Dubernet, F. Daniel, A. Grosjean, C. Y. Lin, Astron. Astroph. 497, 911 (2009).

Herschel is an European Space Agency space observatory with science instruments provided by European-led principal investigator consortia and with important participation from NASA.This work was partially supported by Nederlandse Organisatie voor Wetenschappelijk Onderzoek grant 639.042.404, NSF grant 0707777 and, as part of the NASA Herschel HIFI guaranteed time program, by NASA. The data presented here are archived at the Herschel Science Archive, http://archives.esac.esa.int/hda/ui/, under OBSID 1342198337 and 1342201585. 